\title{
OTIMIZAÇÃO DA EXTRAÇÃO DOS TOCOFERÓIS A PARTIR DO DESTILADO DA DESODORIZAÇÃO DO ÓLEO DE SOJA
}

\author{
MARIA CELIA DE OLIVEIRA HAULY \\ JOSIANI ROMANI RABASSI ${ }^{2}$ \\ SUELY M. OBARA DOI' \\ GENI VARÉA PEREIRA ${ }^{1}$
}

HAULY, M. C. O.; RABASSI, J. R.; DOI, S. M. O.; PEREIRA, G. V. Otimização da extração dos tocoferóis a partir do destilado da desodorização do óleo de soja. Semina: Ci. Biol. Saúde, Londrina, v. 20/21, n. 2, p. 25-31, jun. 1999/2000.

\begin{abstract}
RESUMO: As indústrias de refinação de óleos vegetais geram entre 0,10 e 0,15\% de um subproduto denominado de destilado da desodorização do óleo de soja (DDOS), a partir do óleo bruto total submetido à desodorização. O destilado é um subproduto rico em ácidos graxos livres e lipideos insaponificáveis, entre eles esteróis e tocoferóis. Os tocoferóis apresentam grande atividade de vitamina $E$ e também antioxidante. Em função destas propriedades podem ser utilizadas na formulação de produtos farmacêuticos e cosméticos; como suplemento de ração animal, especialmente par aves, e como aditivo na indústria de alimentos. O presente estudo tem por objetivo otimizar a extração dos tocoferóis presentes no destilado da desodorização do óleo de soja, através da metodologia de superfície de resposta empregando-se experimento fatorial incompleto $3^{3}$. O teor de tocoferol e o rendimento da fração insaponificável foram determinados através de espectrofotometria e gravimetria, respectivamente. A metodologia de superficie de resposta definiu a concentração de $\mathrm{KOH}$ como a variável mais importante devendo ser maior que $50 \%$ e menor que $60 \%$; os volumes de éter mais adequados para obtenção de tocoferóis e extração da fração insaponificável foram $200 \mathrm{~mL}$ e $400 \mathrm{~mL}$ respectivamente; o tempo de saponificação deve ser fixado em 20 minutos.
\end{abstract}

PALAVRAS-CHAVE: Tocoferóis; extração de tocoferóis; destilado da desodorização do óleo de soja; metodologia de superficie de resposta.

\section{INTRODUÇÃO}

Para obtenção de óleos vegetais comestíveis, com caracteristicas organolépticas adequadas para envasamento, consumo direto, ou como ingredientes na produção de alimentos, o óleo bruto é submetido a vários procedimentos, entre eles a desodorização. Esse processo consiste no aquecimento do óleo bruto a cerca de $260^{\circ} \mathrm{C} \mathrm{com}$ retirada, por destilação, de compostos voláteis responsáveis pelos odores desagradáveis, como aldeídos, cetonas, ácidos graxos oxidados, hidrocarbonetos, ácidos graxos de cadeia média e curta, peróxidos e glicerídeos parciais, removendo também matéria insaponificável menos volátil como, esteróis e tocoferóis (Gosh \& Bhattacharyya, 1996). Devido à volatilidade vários compostos são perdidos durante o processo de desodorização do óleo bruto, formando quantidades significativas de um produto denominado de destilado da desodorização do óleo de vegetais (DDO). Esse subproduto corresponde à 0,1 a 0,4
$\%$ do peso original do óleo, contendo entre 0,8 a $10 \%$ de tocoferóis (Almeida et al.,1994).

As indústrias químicas, farmacêuticas, cosméticas e alimentícias têm se interessado pelo DDOS devido à riqueza de seus componentes (Nogala-Katucka et al.,1993). Entre estes, os tocoferóis são componentes interessantes devido à sua atividade de Vitamina $E$ e na sua possivel aplicação como antioxidante (Lee et al.,1991; Snyder \& Know, 1984).

Considerando o potencial brasileiro na produção agrícola de soja, em média 31 milhões de toneladas em grãos (Companhia Nacional de Abastecimento,1998), na industrialização de sementes vegetais oleaginosas, e a necessidade do desenvolvimento de tecnologia nacional para o fornecimento interno de tocoferóis com aplicação em diferentes setores industriais, o presente trabalho tem como objetivo otimizar, atraves da metodologia de superficie de resposta, as condições de saponificação e extração dos tocoferóis, viabilizando o aproveitamento do DDO

\footnotetext{
${ }_{1}^{1}$ Docentes do Departamento de Bioquímica da Universidade Estadual de Londrina. E-mail:biq@uel.br Cx. Postal 6001, CEP 86051970 - Londrina - PR

${ }^{2}$ Aluna do curso de Especialização em Bioquímica Aplicada - Departamento de Bioquímica, Universidade Estadual de Londrina.
} 
gerado durante o processo de desodorização empregado pelas indústrias de refinação de óleos vegetais.

\section{MATERIAL E MÉTODOS}

Matéria prima: Utilizou-se destilado de derodorização de óleo de soja (DDOS) gerado pelo processo de desodorização do óleo de soja. As amostras foram coletadas na refinaria de óleo da COCAMAR - Maringá - PR.

Saponificação: Os lipídios presentes no DDOS foram submetidos à reação de saponificação conforme Christie et al. (1973) modificado, utilizando-se propilgalato em substituição ao ácido ascórbico (Diziezak, 1986).

Para otimizar as condições de reação de saponificação utilizou-se solução aquosa de $\mathrm{KOH}$ em diferentes concentrações (40; 50 ou $60 \%$ ) e tempo de reação diferentes (20; 35 e 50 minutos).

Ao término da reação foram adicionados 6,5 ; 8,5 ou $10 \mathrm{~mL}$ de solução aquosa de $\mathrm{HCl}$ na proporção $1: 1(\mathrm{~V} / \mathrm{V})$, conforme as respectivas concentrações de $\mathrm{KOH}(40 ; 50$ ou $60 \%$ ).

A solução obtida na reação de saponificação foi transferida para um funil de separação (capacidade de $1000 \mathrm{~mL}$ ) tendo-se o cuidado de retirar todo o material do balão, utilizando-se uma quantidade mínima de éter de petróleo. O restante do éter foi adicionado em 3 etapas sucessivas seguidas de agitação para separar a fração insaponificável. A fração inferior contendo o sabão foi descartada, e a insaponificável foi lavada com água destilada em quantidade suficiente para neutralizar a base ou seja, até reação negativa com fenolftaleína. No caso de haver formação de uma fase intermediária (leitosa) entre a fração insaponificável (superior) e a água, esta deve ser coletada e analisada quanto à presença de tocoferóis (AOAC,1995).

O éter foi completamente eliminado em evaporador rotatório, utilizando-se balão de destilação previamente tarado, a fim de determinar gravimetricamente a massa insaponificável.

A análise colorimétrica foi realizada em $534 \mathrm{~nm}$ (AOAC, 1995).

Delineamento estatistico dos experimentos: foi utilizado um delineamento fatorial incompleto $3^{3}$, com 3 variáveis independentes e 3 niveis eqüidistantes de variação, envolvendo 15 unidades experimentais com 3 repetições no ponto central (Cordenunsi et al.,1985).

As variáveis independentes e os respectivos níveis de variação utilizados na reação de saponificação e extração da fração insaponificável estão indicadas na Tabela 1.

As respostas observadas foram teor de tocoferol $\left(Y_{1}\right)$ e rendimento da fração insaponificável $\left(Y_{2}\right)$.

Tabela 1 - Variáveis independentes e niveis de variação do delineamento estatístico para o desenvolvimento experimental da reação de saponificação e extração da fração insaponificável.

\begin{tabular}{lrrr}
\hline & & \multicolumn{2}{c}{ Niveis de variação } \\
\hline & -1 & 0 & +1 \\
\hline X1 - Volume de éter para extraçäo $(\mathrm{mL})$ & 200 & 300 & 400 \\
X2 - Concentração de KOH para saponificação $(\%)$ & 40 & 50 & 60 \\
X3 - tempo da reação de saponificação (min.) & 20 & 35 & 50 \\
\hline
\end{tabular}

\section{RESULTADOS E DISCUSSÃO}

O teor de tocoferol $\left(Y_{1}\right)$ e o rendimento da fração insaponificável $\left(Y_{2}\right)$ obtido nos diferentes experimentos estão apresentados na Tabela 2.

O experimento $\mathrm{n}^{\circ} 5$ apresentou maior teor de tocoferol $(3,0 \mathrm{~g} / 100 \mathrm{~g}$ de lipídio insaponificável). Neste experimento foram utilizados $\mathrm{KOH} 50 \%$; tempo de saponificação de $20 \mathrm{~min}$. e $400 \mathrm{~mL}$ de éter para extração da fração insaponificável.

Motta \& Araújo (1991) encontraram 10,7\% e $16,0 \%$ de tocoferol em amostras de diferentes DDOS o que demonstra a variação do teor de tocoferol conforme a natureza e procedência do subproduto.
Pode-se observar nos experimentos ( $\mathrm{n}^{\circ} 1 ; 2 ; 9 \mathrm{e}$ 11) onde utilizou-se $\mathrm{KOH} 40 \%$ na reação de saponificação, que não foi extraída a fração insaponificável e consequentemente, não foi detectado tocoferol, independentemente do tempo de reação e do volume de éter utilizados na extração da fração insaponificável, mostrando a marcada influência da concentração adequada de $\mathrm{KOH}$ para obtenção de tocoferóis.

Com relação ao rendimento da fração insaponificável $\left(\mathrm{Y}_{2}\right)$, a Tabela 2 mostra que o experimento $n^{\circ} 6$ apresentou maior rendimento $(28,7$ $\mathrm{g} / 100 \mathrm{~g}$ de DDOS). No caso, as condições utilizadas forma $200 \mathrm{~mL}$ de éter; $\mathrm{KOH} 50 \%$ e tempo de 
Tabela 2 - Teor de tocoferol $\left(Y_{1}\right)$ e rendimento da fração insaponificável $\left(Y_{2}\right)$.

\begin{tabular}{cccccc}
\hline $\begin{array}{c}N^{0} \text { dos } \\
\text { experimentos }\end{array}$ & $X_{1}(\mathrm{~mL})$ & $X_{2}(\%)$ & $X_{3}(\min )$. & & $Y_{1}{ }^{*}$ \\
\hline 1 & 200 & 40 & 35 & 0,00 & 0,00 \\
2 & 400 & 40 & 35 & 0,00 & 0,00 \\
3 & 200 & 60 & 35 & 2,7735 & 11,8627 \\
4 & 400 & 60 & 35 & 1,4429 & 22,0389 \\
5 & 200 & 50 & 20 & 3,0015 & 13,3792 \\
6 & 400 & 50 & 20 & 1,1512 & 28,6986 \\
7 & 200 & 50 & 50 & 1,4623 & 15,1977 \\
8 & 400 & 50 & 50 & 1,4650 & 25,6783 \\
9 & 300 & 40 & 20 & 0,00 & 0,00 \\
10 & 300 & 60 & 20 & 1,2625 & 20,5779 \\
11 & 300 & 40 & 50 & 0,00 & 0,00 \\
12 & 300 & 60 & 50 & 1,6680 & 16,1369 \\
13 & 300 & 50 & 35 & 1,9126 & 20,5352 \\
14 & 300 & 50 & 35 & 1,3680 & 18,2211 \\
15 & 300 & 50 & 35 & 2,1632 & 21,0029 \\
\hline
\end{tabular}

* teor de tocoferol em g/100 g de lipideo insaponificável.

** rendimento da fração insaponificável emg/100 g de DDOS

saponificação de 20 minutos. Pode-se dizer que $\mathrm{KOH} 50 \%$, de modo semelhante ao teor de tocoferol $\left(Y_{1}\right)$, exerceu grande influência na extração insaponificável $\left(Y_{2}\right)$.

Comparando-se os experimentos $\mathrm{n}^{\circ} 5$ e $\mathrm{n}^{\circ} 6$ os quais apresentaram maior teor de tocoferol e maior rendimento da fração insaponificável respectivamente, podemos observar que em ambos, a concentração de $\mathrm{KOH}$ foi de $50 \%$ e o tempo de saponificação de 20 minutos.

A análise de variância para o teor de tocoferol $\left(Y_{1}\right)$ indicada na Tabela 3, mostra que $58,59 \%$ da variação é explicada pelo efeito linear das variáveis independentes, enquanto $24,36 \%$ é explicada pelo efeito quadrático. Entretanto, somente 10,25\% é explicada pelo efeito de interação entre as variáveis concentração de $\mathrm{KOH}$, volume de éter e tempo de saponificação.

O volume do coeficiente de determinação total $R^{2}$ igual a 0,9320 , significa que $93,20 \%$ da variação em torno da média é explicada pela análise de regressão.
Portanto, pode-se dizer que o modelo estatístico se ajustou aos dados experimentais.

Através da análise de variância apresentada na Tabela 3 foi possivel analisar os niveis de significância. Observa-se que os efeitos linear, quadrático e regressão total foram significativos ao nivel de $5 \%$ de probabilidade.

A Tabela 4 mostra a significância das variáveis independentes quanto aos efeitos linear, quadrático e de interação das mesma sobre o teor de tocoferol $\left(Y_{1}\right)$. Pode-se observar que somente os efeitos lineares de $X_{1}(0,0455)$ e $X_{2}(0,0019)$ e o efeito quadrático de $\mathrm{X}_{2}(0,0095)$ foram significativos ao nível $5 \%$ de probabilidade.

É importante ressaltar que a variável $\mathrm{X}_{3}$, ou seja, tempo de saponificação, não foi significativa (ao nivel de $5 \%$ de probabilidade) mostrando que esta variável não influenciou na obtenção de tocoferol nas condições testadas. Portanto, recomenda-se a utilização do menor tempo de saponificação (20 minutos) para viabilização industrial e econômica.

Tabela 3 - Análise de variância para o teor de tocoferol $\left(Y_{1}\right)$.

\begin{tabular}{|c|c|c|c|c|}
\hline Fonte de variação & $\begin{array}{l}\text { Graus de } \\
\text { liberdade }\end{array}$ & $\begin{array}{l}\text { Soma dos } \\
\text { quadrados }\end{array}$ & Quadrado médio & $\begin{array}{c}\text { Probabilidade } \\
\text { (Teste F) }\end{array}$ \\
\hline Linear & 3 & 7,106 & 0,5859 & $0,0068^{\star}$ \\
\hline Quadrático & 3 & 3,2057 & 0,2436 & $0,0417^{*}$ \\
\hline Interação & 3 & 1,3491 & 0,1025 & 0,1729 \\
\hline Regressão total & 9 & 12,2654 & 0,9320 & $0,0189^{\star}$ \\
\hline Erro experimental & 2 & 0,3261 & 0,1630 & --.-- \\
\hline $\begin{array}{l}\text { Coeficiente de deter } \\
\text { Coeficiente de varia }\end{array}$ & $\begin{array}{l}\text { ção }\left(R^{2}\right) \text {. } \\
\text { C. } V \text {. }\end{array}$ & & $\begin{array}{r}0,9320 \\
32,2889\end{array}$ & \\
\hline
\end{tabular}

${ }^{*}$ Significativo ao nivel de $5 \%$ de probabilidade $(p<0,05)$ 
Foi verificado que o efeito de interação das variàveis independentes não exerceram influência significativa na obtenção de tocoferol.

A Figura 1 mostra o mapa de contorno obtido para otimizar as condições referentes à concentração de $\mathrm{KOH}$ e volume de éter, para obtenção de maior teor de tocoferol, mantendo-se fixo o tempo de reação de saponificação em 20 minutos. Isto porque conforme demonstrado anteriormente este parâmetro não interfere significativamente na resposta avaliada. Os melhores valores de tocoferóis compreendidos na faixa de 3,0 a $3,75 \mathrm{~g} / 100 \mathrm{~g}$ de lipídios insaponificáveis representados no gráfico pelo símbolo? foram obtidos utilizando-se $\mathrm{KOH}$ numa concentração maior que $50 \%$ e menor que $60 \%$. Os valores codificados são 0,4 a 0,9 , ou seja, $54 \%$ a $59 \%$ para o álcali e $-1,0$ para o éter, ou seja, $200 \mathrm{~mL}$, podem ser vistos na Figura 1. Considerando-se que a região ótima indicada na Figura 1 não define exatamente qual a concentração de $\mathrm{KOH}$ deve ser utilizada para obtenção de maior teor de tocoferol, sugere-se um estudo de otimização utilizando-se $200 \mathrm{~mL}$ de éter como ponto central da futura investigação.

Considerando que através da Tabela 3 , foi verificado que a concentração de $\mathrm{KOH}\left(X_{2}\right)$, foi a variável independente de maior significância,

Tabela 4 - Significância das variáveis independentes na obtenção de tocoferol $\left(Y_{1}\right)$.

\begin{tabular}{|c|c|}
\hline Efeito das Variáveis Independentes & Probabilidade \\
\hline \multicolumn{2}{|l|}{ Linear } \\
\hline $\mathrm{X}_{1}$ (volume de éter, $\mathrm{mL}$ ) & $0,0455^{\star}$ \\
\hline $\mathrm{X}_{2}$ (concentração de $\mathrm{KOH}, \%$ ) & $0,0019^{*}$ \\
\hline$X_{3}$ (tempo de saponificação, min.) & 0,5286 \\
\hline \multicolumn{2}{|l|}{ Quadrático } \\
\hline$x_{1} \cdot x_{1}$ & $0,5 \overline{575}$ \\
\hline$x_{2} \cdot x_{2}$ & $0,0095^{*}$ \\
\hline$x_{3} \cdot x_{3}$ & 0,4470 \\
\hline \multicolumn{2}{|l|}{ Interação } \\
\hline$X_{2} \cdot X_{1}$ & 0,1769 \\
\hline$x_{3} \cdot x_{1}$ & 0,0793 \\
\hline$X_{3} \cdot X_{2}$ & 0,6486 \\
\hline
\end{tabular}

${ }^{\star}$ Significativo ao nivel de $5 \%$ de probabilidade $(P<0,05)$

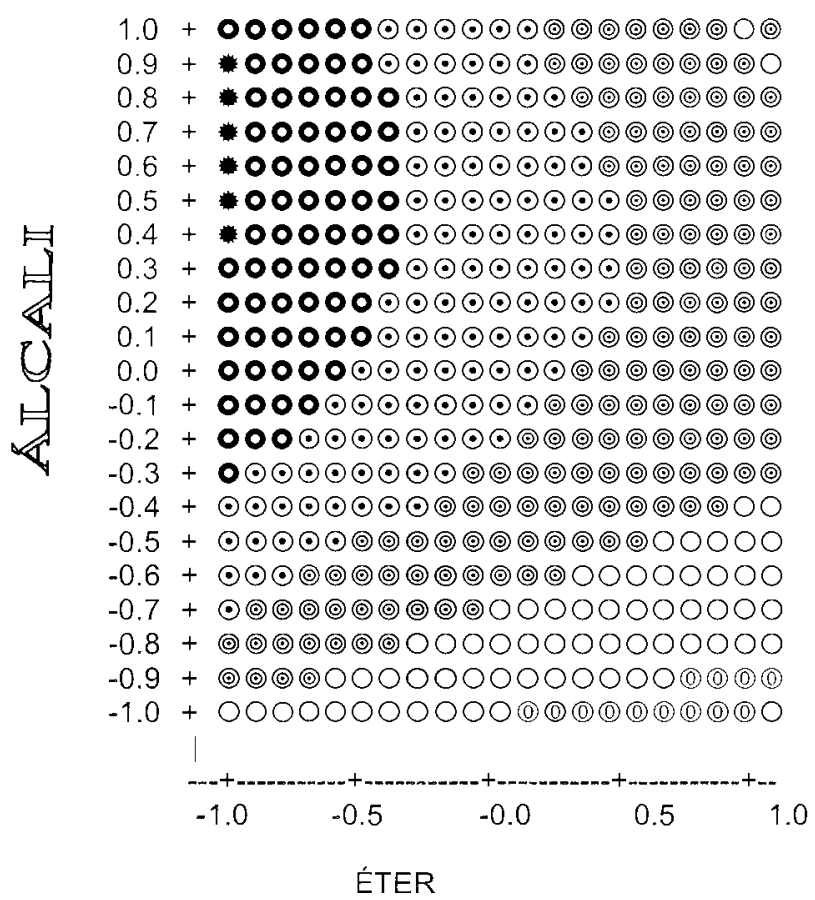

Figura 1 - Mapa de contorno obtido referente a otimização da concentração de $\mathrm{KOH}(\%)$ e volume de éter ( $\mathrm{mL}$ ) para obtenção de maior teor de tocoferol. 
sugere-se que nos próximos experimentos a serem realizados, este parâmetro seja a variável $\left(x_{1}\right)$ e conseqüentemente, o volume de éter seja $\left(x_{2}\right)$. O tempo de saponificação poderá ser fixado em 20 minutos.

O valor máximo encontrado para o teor de tocoferol $(3,0 \mathrm{~g} \%)$ foi relativamente baixo, entretanto, é importante destacar que a amostra de DDOS utilizada nos experimentos foi obtida de única refinaria e como demonstrado por Almeida et al. (1994) existe grande variação na composição química das amostras conforme a procedência. $O$ autor analisou através de CLAE, o teor de tocoferol em 3 diferentes amostras de DDOS e os valores obtidos foram $8,60 \mathrm{~g} / 100 \mathrm{~g}$ de DDOS; $9,30 \mathrm{~g} / 100 \mathrm{~g}$ DDOS e $3,70 \mathrm{~g} / 100 \mathrm{~g}$ de DDOS. Os resultados mostram a grande variação na composição química de amostras diferentes.

Através de cromatografia gasosa foi encontrado $4,8 \%$ de tocoferol em destilado da desodorização do óleo de girassol (Gosh \& Bhattacharyya, 1996). Este resultado, relativamente baixo, confirma que a fonte de tocoferol explica, em grande parte, a variação do teor de tocoferol.

A análise de variância para o rendimento da fração insaponificável $\left(Y_{2}\right)$ encontra-se na Tabela 5 . Pode-se verificar que $57,84 \%$ da variação é explicada pelo efeito linear das variáveis independentes; 36,74 $\%$ da variação é explicada pelo efeito quadrático e somente $2,69 \%$ refere-se ao efeito de interação entre as variáveis independentes $X_{1}, X_{2}$ e $X_{3}$ que são respectivamente, volume de éter, concentração de $\mathrm{KOH}$ e tempo de saponificação.

Relacionado aos niveis de significância, podemos verificar através da Tabela 6 que, os efeitos linear, quadrático e regressão total foram significativos ao nivel de $5 \%$ de probabilidade. Entretanto o efeito de interação não foi significativo.

Na Tabela 6 verifica-se que somente os efeitos lineares de $X_{1}(0,0055)$ e $X_{2}(0,0003)$ foram significativos para o rendimento da fração insaponificável. Observa-se pelo valor apresentando quanto à probabilidade que a variável $X_{2}$ (concentração de $\mathrm{KOH}$ ) é mais importante que a variável $X_{1}$ (volume de éter). Somente o efeito quadrático de $X_{2}(0,0005)$ foi significativo reforçando a influência desta variável para obtençäo de maior rendimento da fração insaponificável. Por outro lado, a variável independente $X_{3}$ (tempo de saponificação) não foi significativa, ao nível de $5 \%$ de probabilidade, quanto aos efeitos linear e quadrático, mostrando que esta variável não influencia no rendimento da fração insaponificável. Portanto, sugere-se a utilização do menor tempo, ou seja, 20 minutos que é mais economicamente viável. Pode-se observar neste quadro que de modo semelhante ao processo de obtenção de tocoferol $\left(Y_{1}\right)$, o efeito de interação entre as variáveis independentes não influenciou significativamente no rendimento da fração insaponificável.

A Figura 2 mostra o mapa de contorno obtido para otimizar as condições experimentais referentes à concentração de $\mathrm{KOH}$ e volume de éter para obtenção de maior rendimento da fração insaponificável $\left(\mathrm{Y}_{2}\right)$. O tempo de saponificação foi fixado em 20 minutos, pois como demonstrado anteriormente, esta variável não influencia significativamente no processo. Os maiores valores de $Y_{2}$ (30 a $37 \mathrm{~g} / 100 \mathrm{~g}$ de DDOS) representados na Figura 2 pelo simbolo $\Theta$, foram obtidos utilizandose os valores codificados de 0,4 a 0,7 , correspondente a $54 \%$ a $57 \%$ de álcali e 1,0 para o éter, equivalente a $400 \mathrm{~mL}$.

Como a concentração exata de $\mathrm{KOH}$ não está definida na região ótima da Figura 2, recomenda-se fazer um novo estudo de otimização das condições experimentais para obtenção de um maior rendimento da fração insaponificável, utilizando-se $\mathrm{KOH}$ na faixa de 50 a $58 \%$ tendo como ponto central $54 \%$.

Tabela 5 - Análise de variância para o rendimento da fração insaponificável $\left(Y_{2}\right)$.

\begin{tabular}{|c|c|c|c|c|}
\hline Fonte de variação & $\begin{array}{l}\text { Graus de } \\
\text { liberdade }\end{array}$ & $\begin{array}{l}\text { Soma dos } \\
\text { quadrados }\end{array}$ & Quadrado médio & $\begin{array}{c}\text { Probabilidade } \\
\text { (Teste F) }\end{array}$ \\
\hline Linear & 3 & 788,5784 & 0,5784 & $0,0009^{\star}$ \\
\hline Quadrático & 3 & 500,8194 & 0,3674 & $0,0025^{*}$ \\
\hline Interação & 3 & 36,6420 & 0,0269 & 0,2932 \\
\hline Regressão total & 9 & 1326,0362 & 0,9727 & $0,0021^{*}$ \\
\hline Erro experimental & 2 & 4,4285 & 2,2142 & $----\cdots-$ \\
\hline \multicolumn{3}{|c|}{ Coeficiente de determinação $\left(\mathrm{R}^{2}\right) \ldots \ldots \ldots \ldots \ldots \ldots \ldots \ldots \ldots \ldots \ldots \ldots \ldots \ldots$} & 0,9727 & \\
\hline \multicolumn{3}{|c|}{ 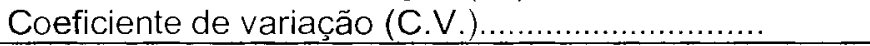 } & 19,2020 & \\
\hline
\end{tabular}

${ }^{*}$ Significativo ao nivel de $5 \%$ de probabilidade $(p<0,05)$ 
Tabela 6 - Significância das variáveis independentes par o rendimento da fração insaponificável $\left(Y_{2}\right)$.

\begin{tabular}{|c|c|}
\hline Efeito das Variáveis Independentes & Probabilidade \\
\hline \multicolumn{2}{|l|}{ Linear } \\
\hline $\mathrm{X}_{1}$ (volume de éter, $\mathrm{mL}$ ) & $0,0055^{\star}$ \\
\hline $\mathrm{X}_{2}$ (concentração de $\mathrm{KOH}, \%$ ) & $0,0003^{*}$ \\
\hline$X_{3}$ (tempo de saponificação, min.) & 0,4979 \\
\hline \multicolumn{2}{|l|}{ Quadrático } \\
\hline$X_{1} \cdot X_{1}$ & 0,9704 \\
\hline $\mathrm{X}_{2} \cdot \mathrm{X}_{2}$ & $0,0005^{\star}$ \\
\hline$x_{3} \cdot x_{3}$ & 0,6166 \\
\hline \multicolumn{2}{|l|}{ Interação } \\
\hline $\mathrm{X}_{2} \cdot \mathrm{X}_{1}$ & 0,1216 \\
\hline$x_{3} \cdot x_{1}$ & 0,4160 \\
\hline $\mathrm{X}_{3} \cdot \mathrm{X}_{2}$ & 0,4531 \\
\hline
\end{tabular}

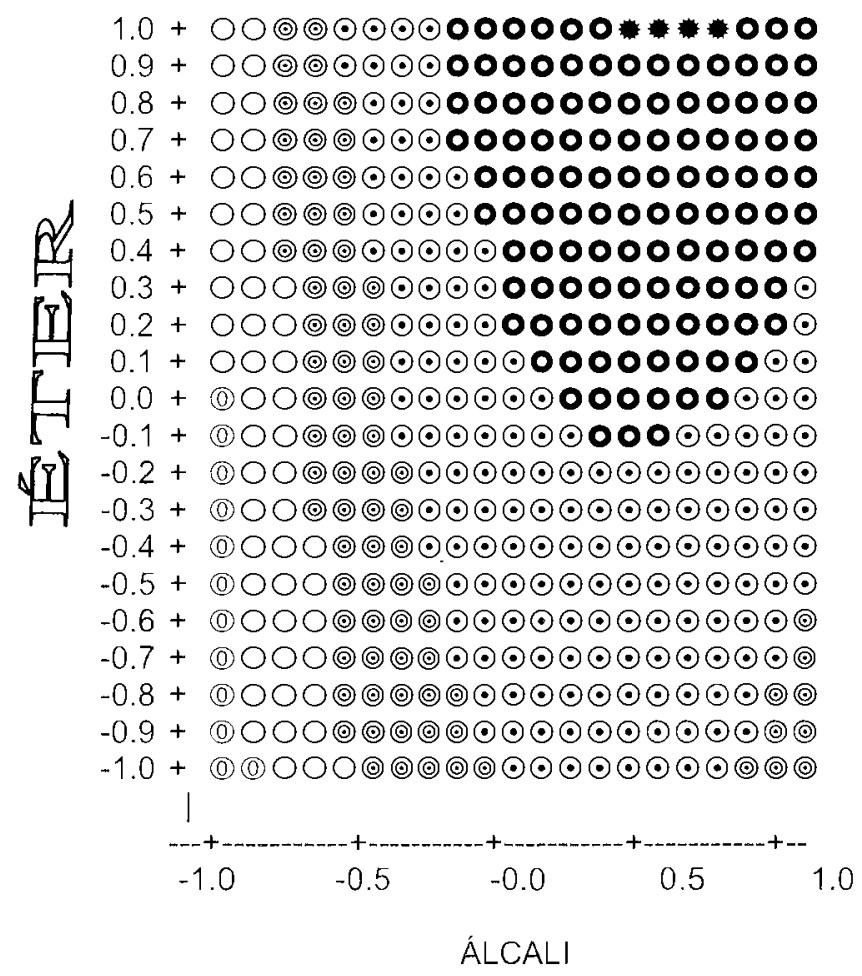

Figura 2 - Mapa de contorno referente à otimização da concentração de $\mathrm{KOH}$ e volume de éter para obtenção de maior rendimento da fração insaponificável.

\section{CONCLUSÕES}

- A Metodologia de superfície de resposta mostrou quea concentração de KOHéavariável independente mais importante para obtenção de tocoferol e fração insaponificável. Entretanto, o tempo de saponificação não influenciou significativamente nas condições testadas, podendo ser fixado em 20 minutos.

- A otimização para obtenção de tocoferol, a partir do destilado da desodorização do óleo de soja, mostrou que a concentração de $\mathrm{KOH}$ maior que $50 \%$ e menor que $60 \% ; 200 \mathrm{~mL}$ de éter e tempo de saponificação de 20 minutos foram os mais adequados.

- Aotimização para obtenção da ração insaponificável, a partir do destilado da desodorização do óleo de soja, mostrou que a concentração de $\mathrm{KOH}$ maior que $50 \%$ e menos que $60 \% ; 400 \mathrm{~mL}$ de éter e tempo de saponificação de 20 minutos foram os mais adequados. 
HAULY, M. C. O.; RABASSI, J. R.; DOI, S. M. O.; PEREIRA, G. V. Optimization of tocopherols extraction from soybean oil desodorizer distillate. Semina: Ci. Biol. Saúde, Londrina, v. 20/21, n. 2, p. 25-31, jun. 1999/2000.

ABSTRACT: A refinery subproduct named of soybean Oil Deodorizer distillate (SODD) is rich in tocopherols. In order to study the best conditions to extract the tocopherols an incomplete factorial planning was developed. Three variables were studied: volume of ether (200 to $400 \mathrm{~mL}$ ). $\mathrm{KOH}$ concentration (40 to $60 \%$ ) and saponification time (20 to 50 minutes). Tocopherols were determined by spectrofotometry at $534 \mathrm{~nm}$. The best quantity of tocopherols was $3 \mathrm{~g} / 100 \mathrm{~g}$ from unsaponifiable matter and $28,3 \mathrm{~g} / 100 \mathrm{~g}$ of SODD for the rate of unsaponifiable matter. The $\mathrm{KOH}$ concentration was the most important variable which must be higher than $50 \%$ and less than $60 \%$. The best ether volumes were $200 \mathrm{~mL}$ and $400 \mathrm{~mL}$ in order to get highest quantity of tocopherols and unsaponifiable matter respectively. The saponification time can be fixed in 20 minutes.

KEYWORDS: TOCopherols; tocopherols extraction; soybean oil distillate; response surface methodology.

\section{REFERÊNCIAS BIBLIOGRÁFICAS}

ALMEIDA. M.E.M.; GUSMAN, E.C.; CARVALHO,P.R.N.; RUSIG O. Avaliação de destilado da desodorização do óleo de soja para a extração da vitamina E. Arquivo de Biologia e Tecnologia. Curitiba, v.37, n.4, p.1003-1011, dez., 1994.

ASSOCIATION OF OFFICIAL ANALYTICAL CHEMISTS (AOAC). Official Methods of Analyses. Arlington: AOAC, 1995. Cap. 45, p. 30-33

CHRISTIE, A.A.; DEAN, A.C.; MILLBURN, B.A. The determinationof vitamin $E$ in food by colorimetry and gasliquid chromatography. Analyst, v.98, p.161-167, Mar. 1973.

COMPANHIA NACIONAL DE ABASTECIMENTO. Indicadores da Agropecuária. v.1, n.7, p.38-47, dez./jan. 1998.

CORDENUNSI, B.R.; DA SILVA, R.S.F.; SRIVASTAVA, K.C; FABRE SANCHES, S.; PERRE, M.A. Mathematical model for the alcoholic fermetnation in bath culture: comparison between complete and incomplete factorial $\left(3^{3}\right)$ designs. Journal of Biotechnology. Amsterdan, v.2, p.1-12, 1985.

DIZIEZAK, J. D. (Ed.) Preservatives: antioxidants the ultimate answer to oxidation. Food Technology, p.94-102, Sept., 1986.
GOSH, S.: BHATTACHARYYA, D.K. Isolation of tocopheroland strol concentrate from sunflower oil deodorizer distilate. The Joumal of American Oil Chemists Society, v.73, n.10, Oct., 1996.

LEE, H.; CHUNG, B.H.; PARK, Y.H. Concentration of tocopherols from soybean sludge by supercritical carbon dioxide. The Joumal of American Oil Chemists Society. v..68, n.8, p.571573, Aug., 1991

MOTTA, S.; ARAÚJO, J. M.A. Concentração de vitamina E a partir de destilado de óleo de soja. Arquivo de Biologia e Tecnologia. Curitiba, v.34, n.2, p.217-226, jun., 1991.

NOGALA-KATUCKA, M.; GOGOLELWSKI, M.; SWIATKIEICZ, E. Changes in the composition of tocopherols and fatty acids in postdeodorisation condensates during refining of oils. Fat Science Technology. n.4, 1993.

SNYDER, H.E.; KNOW. T.W. Soybean Utilization. New York: Avi, $1987.346 \mathrm{p}$ 